2003. Vol. 10. - P. 306 - 314.

9. De Carolis S., Fatigante G., Ferrazzani S., Trivellini C., De SantisL, Mancuso S., and Caruso A. Uterine myomectomy in pregnant women.//Fetal Diagn Ther, 2001.; 16 (2): 116-9.

10. Walker C. L., Cesen-Cummings K., Houle C., Baird D., Barrett J. C., Davis B. Protective effect of pregnancy for development of uterine leiomyoma. // Carcinogenesis, 2001, Vol. 22, No. 12, P. 2049-2052.

DOI: 10.22448/AMJ.2016.15-16.90-91

UDC 796

LOCOMOTIVE ACTIVITY AND WEIGHT

Mironov F.S., Moseikina V.V.

Amur State Medical Academy, Blagoveshchensk, Russia

Abstract The aim of the study is to analyze interrelation of the Amur region women's weights to their levels of locomotive activity and age.

Key words: physical activity, weight, blood pressure.

There is an opinion, that it is typical for a mature person to gain weight. However, it is deeply flawed opinion because person's weight during the whole life should remain at about the same level as it has established in adolescence. Young people and teenagers do not usually eat less than adults, but they are in good shape because the majority of them are active and do sports or have other physical activities. A prevalent cause of overweight among middle-aged people is loss of physical activity. What is more, it can be explained by the fact that a person's appetite remains the same, even when the person spends significantly less calories. The body has no way to get rid of surplus and gradually accumulates it as fat. As a result, there is the deposition of excess weight, provoked by sedentary lifestyle.

The obesity not only doesn't make a man more attractive, but also has a harmful effect on his health and general well-being. Physical inactivity damages the normal activity of the body, people with overweight suffer from physical and mental stress and they are more susceptible to various diseases. The most favorable way out of this situation is to maintain an active lifestyle, which contributes to the normalization of the body weight. According to the index of Quetele the optimum weight is 350-400 g of the body weight per one centimeter of man's growth, 325-375 g for one centimeter of woman's growth.

One of the important conditions for the maintenance of normal weight is balancing the incoming energy with its spending. Moreover, the use of moderate physical activity is inherent for the organism. Physical activity is essential for the metabolism. During brief physical activity consumption of the energy, which is drawed primary upon ATP and then from glucose, is rapidly increasing in the muscles. During the long muscular work the use of fatty acids increases.

In order to maintain metabolism and muscle tone at an acceptable level, there are effective physical exercises from morning hygienic gymnastics, jogging, cycling till popular nowadays fitness, pilates, yoga practices and others forms of activity.

It is advisable to have at least a part of the motor activity with the emotional basis. During any collective physical exercises the larger amount of catecholamines is giving out, than during the monotonous physical exercises (catecholamines accelerate the combustion of reserved fat and glycogen). Isometric exercises aren't suitable for fat people (the muscle is strained, but it doesn't contract rhythmically), because muscle mass increases, but body weight reduces slightly.

We have conducted the research, which has carried out the interrelation of physical activity to weight and age, additionally, we have noted the state of the cardiovascular system among people of different age categories.

Young age participant's indicators:

1. girl, 19 years old: $169 \mathrm{~cm}, 49 \mathrm{~kg}$, blood pressure: $87 / 57,12.450$ steps per day;

2. girl, 22 years old: $169 \mathrm{~cm}, 56 \mathrm{~kg}$, blood pressure 100/65, 11,895 steps a day;

Middle age participant's indicators:

1.woman, 39 years old: $170 \mathrm{~cm}, 80 \mathrm{~kg}$, blood pressure: $120 / 80,5187$ steps a day;

Old age participant's indicators:

1. woman, 59 years old: $160 \mathrm{~cm}, 72 \mathrm{~kg}$, blood pressure: $90 / 66,4120$ steps a day;

2. woman, 64 years old, $164 \mathrm{~cm}, 95 \mathrm{~kg}$, blood pressure: $140 / 90,3980$ steps a day.

It is important to highlight a couple of issues, which can not be ignored during any physical exercises. Before starting a training, you should have time for a preliminary «warming» of the whole body with the help of some simple exercises. Pay attention to the strengthening of muscles and ligaments of the joints, muscles of the abdomen, back and chest. All exercises should be combined with the correct breathing: inhale slowly and 
deeply, duration of the exhale should be longer then inhale. The intensity of the exercises should be adjusted according to human's physical state and overall well-being.

Don't seek to "round up" the weight as soon as possible. Loss of 4-5 kg in two or three months is considered to be acceptable to the body, but more dramatic weight loss can give undesirable effect. It is important to remember that the measure should present even in the active lifestyle, do not misuse the amount of exercises.

DOI: 10.22448/AMJ.2016.15-16.91-92

\section{УДК 616.831-005.4:616.8-07 \\ THE POSSIBILITIES OF NON-PHARMACOLOGICAL CORRECTION OF ENDOTHELIAL DYSFUNCTION IN THE ACUTE PERIOD OF ISCHEMIC STROKE \\ Molchanova E.E.}

\section{Amur State Medical Academy, Blagoveshchensk, Russia}

Abstract The level of plasma endothelin-1 was studied depending on sex, age, severity of the disease and of the therapy by conducting a survey of 43 patients with acute ischemic stroke, carried out in the primary vascular center. At the time of admission an increase in plasma concentrations of endothelin-1 to an average of $0,97 \pm 0,23 \mathrm{fmol} / \mathrm{ml}$ was revealed. The maximum blood concentration of endothelin-1 was recorded among patients with moderate and severe neurological deficits and those over the age of 60 years old. Analysis of endothelin-1 level after the course of early rehabilitation has shown that the use of acupuncture leads to significantly greater reduction of this index after 2 weeks of treatment. Therefore, combination of pharmaceuticals effects with the early application of acupuncture techniques greatly reduces the severity of endothelial dysfunction and contributes to the successful rehabilitation of the patients.

Key words: ischemic stroke, endothelial dysfunction, endothelin-1

Stroke takes one of the first places as a cause of death and is major cause of disability. [2] Thus, a cerebral catastrophe entails heavy moral, social and economic consequences. Currently, the major importance is given to the study of the acute cerebral ischemia pathophysiology and the development of effective methods for correction of detected changes. Scientific researches of the last decade, that have been made in our country and abroad, suggest that endothelial dysfunction (ED) is one of the universal mechanisms of the pathogenesis of atherosclerosis, hypertension, coronary heart disease and may be an independent predictor of coronary and cerebral accidents $[1,3,6,7]$. Accumulated evidence of the role of endothelium in the pathogenesis of cardiovascular diseases has led to the concept of the endothelium formation as a target for prevention and treatment of cerebrovascular diseases. ED can be defined as an imbalance between relaxing and constrictor factors [8]. Development of these disorders is mainly caused by a decrease in biological activity of the primary vasodilator - NO, and increase of vasoconstrictor endothelin-1, which may be one of the pathogenesis of ischemic stroke [1,9]. There is no doubt that the positive impact on the ED, cerebral and cardiac hemodynamics - is one of the most important parts of the treatment of patients with ischemic stroke.

The aim of our study was:

1.To study of the level of endothelin-1 among patients with acute ischemic stroke according to gender, age, and disease severity

2.To asses the possible impact of correcting reflexology techniques on the background of basic treatment on the severity of ED in patients with acute ischemic stroke in primary vascular center.

Materials and methods. We conducted a survey of 43 patients aged from 43 to 78 years old (mean age $63,3 \pm 2,2)$, who were are treated in primary vascular center of Blagoveshchensk. All patients received standard basic therapy. The study group included 30 patients whose standard medical therapy was optimized with different acupuncture techniques. Reflexology included corporal acupuncture, scalp acupuncture [4], ear acupuncture, dynamic electroneurostimulation [5]. The comparison group consisted of 13 people who received only standard therapy. Examined groups were comparable in terms of age, sex, severity of neurological deficit, pathogenic variants of stroke and comorbidities. Both groups consisted predominantly of women $(66.7 \%$ in the main group, and $61.5 \%$ - in the comparison group). In all cases, the diagnosis of ischemic stroke was established in the hospital based on anamnestic data, clinical features and neuroimaging (CT or MRI of the brain). Evaluation of neurological deficit was based on the results of clinical examination and complemented with NIHSS score (National Institute of Health Stroke Scale). The total score on the NIHSS scale of less than 6 is considered as an easy neurological deficit, the range of 7-12 points corresponds to moderate and more than 14 points - severe neurological deficit [10]. At the beginning of therapy, the mean values of the total score on NIHSS scale was $6,73 \pm 0,52$ points in the main group and 6,46 $\pm 1,16$ points - in the comparison group. Severe neurological deficit was observed among 2 patients in the primary and 1 - in the comparison group, moderate - among 8 and 3, respectively, easy - 20 in the main and 9 - in the comparison group.

Endothelin-1 level was studied in venous blood plasma by ELISA using production «Biomedica» kit (Austria) before and after treatment (14 days). Blood sampling was performed in the morning. Values of endo- 